\title{
A New Concept in Advice Complexity of Job Shop Scheduling?
}

\section{Monograph}

Author(s):

Wehner, David

Publication date:

2014

Permanent link:

https://doi.org/10.3929/ethz-a-010889183

Rights / license:

In Copyright - Non-Commercial Use Permitted 


\title{
A New Concept in Advice Complexity of Job Shop Scheduling ${ }^{\star}$
}

\author{
David Wehner \\ ETH Zürich, Rämistrasse 101, 8092 Zürich, Switzerland \\ wehnerd@student.ethz.ch
}

\begin{abstract}
In online scheduling problems, we want to assign jobs to machines while optimizing some given objective function. In the class we study in this paper, we are given a number $m$ of machines and two jobs that both want to use each of the given machines exactly once in some predefined order. Each job consists of $m$ tasks and each task needs to be processed on one particular machine. The objective is to assign the tasks to the machines while minimizing the makespan, i.e., the processing time of the job that takes longer. In our model, the tasks arrive in consecutive time steps and an algorithm must assign a task to a machine without having full knowledge of the order in which the remaining tasks arrive. We study the advice complexity of this problem, which is a tool to measure the amount of information necessary to achieve a certain output quality. A great deal of research has been carried out in this field; however, this paper studies the problem in a new setting. In this setting, the oracle does not know the exact future anymore but only all possible future scenarios and their probabilities. This way, the additional information becomes more realistic. We prove that the problem is more difficult with this oracle than before. Moreover, in job shop scheduling, we provide a lower bound of $1+1 /(6 \sqrt{m})$ on the competitive ratio of any online algorithm with advice and prove an upper bound of $1+1 / \sqrt{m}$ on the competitive ratio of an algorithm from Hromkovič et al. [8].
\end{abstract}

\section{Introduction and Preliminaries}

Knowing the future might be very useful. In hindsight, we often see that our decisions in everyday life were not optimal. If we had known everything we know now, we would have been able to take more adequate actions. Many problems in computer science face the same challenge; the input arrives piecemeal and an algorithm has to make its decisions without knowing the whole input in advance. Such problems are called online (optimization) problems and algorithms for online problems are called online algorithms. After each part of the input, an online algorithm has to produce a part of its final output. Thus, in any time step $i$, an online algorithm cannot revoke decisions made in a time step $j<i$.

As in the real world, where we compare our decisions in hindsight with the knowledge we have now, we judge the quality of an online algorithm by comparing its solution to the solution of an optimal offline algorithm, i.e., an algorithm

\footnotetext{
* This work was partially supported by the SNF grant 200021-141089.
} 
that sees the whole input in advance and produces an optimal output. The competitive ratio is the ratio between the cost of the online algorithm and the cost of an optimal offline algorithm. This well-established approach to measure an online algorithm's performance is called competitive analysis and was introduced by Sleator and Tarjan [12]. In this paper, we study the amount of information necessary to achieve a reasonable competitive ratio for a particular problem; this is done by means of advice complexity, which is introduced later.

We now formally define minimization problems where the whole input is not known in advance but given in consecutive time steps.

Definition 1 (Online Minimization Problem [9]) An online minimization problem consists of a set $\mathcal{I}$ of inputs; for every $I \in \mathcal{I}$, there is a set of feasible outputs $\mathcal{O}(I)$; and there is a cost function cost for all $I \in \mathcal{I}$. Every input $I \in \mathcal{I}$ is a sequence $I=\left(x_{1}, \ldots, x_{n}\right)$ of requests. Every output $O \in \mathcal{O}(I)$ is a sequence $O=\left(y_{1}, \ldots, y_{n}\right)$ of answers to the requests of $I$. The cost function cost: $\mathcal{O}(I) \rightarrow$ $\mathbb{R}$ assigns to every output a real value $\operatorname{cost}(I, O)$. For every $I \in \mathcal{I}$, we call an output $O$ an optimal solution for $I$ if $\operatorname{cost}(I, O)$ is $\min _{O \in \mathcal{O}(I)}\{\operatorname{cost}(I, O)\}$.

Most of the time, we omit the instance $I$ and just write $\operatorname{cost}(O)$ if it is clear from the context to which instance we refer. Algorithms for online minimization problems that construct their output step by step based on the given information about the input are called online algorithms.

Definition 2 (Online Algorithm) Let $I=\left(x_{1}, \ldots, x_{n}\right)$ be an input of an online minimization problem. An online algorithm ALG calculates a feasible output $\operatorname{ALG}(I)=\left(y_{1}, \ldots, y_{n}\right) \in \mathcal{O}(I)$ such that for $i \in\{2, \ldots, n\}, y_{i}$ depends solely on $x_{1}, \ldots, x_{i}$ and on $y_{1}, \ldots, y_{i-1} ; y_{1}$ depends solely on $x_{1}$. The cost of ALG is defined as cost $(\operatorname{Alg}(I))$.

In order to measure how good an online algorithm is, we compare the solutions computed by it to an optimal offline solution, i.e., an optimal solution produced by an algorithm that knows the whole input in advance.

Definition 3 (Competitive Ratio) Let $I=\left(x_{1}, \ldots, x_{n}\right)$ be an input of an online minimization problem and let OPT be an optimal solution for the corresponding offline minimization problem. Let $c \geq 1 \in \mathbb{R}$. An online algorithm ALG has a competitive ratio of $c$ and ALG is called $c$-competitive if $\operatorname{cost}(\operatorname{ALG}(I)) \leq$ $c \cdot \operatorname{cost}(\mathrm{OPT}(I))$.

Note that this is the usual definition of being strictly $c$-competitive; we are not going to distinguish between strictly $c$-competitive and $c$-competitive. When talking about online algorithms, it is common practice to analyze the quality of an online algorithm ALG by introducing an adversary ADV [3,8,10]. ADV constructs input instances for ALG in a pernicious way, i.e., such that the competitive ratio of ALG is as large as possible.

In 2008, Dobrev, Královič, and Pardubská introduced the notion of advice complexity for online problems [4]. They wanted to know what it is exactly 
that makes an online problem harder than the corresponding offline problem. Their model was later refined [5,7]; in this paper, we use the model introduced by Hromkovič et al. [7] and analyzed among others by Komm [9,10,11] as our standard model, which we are going to modify.

The standard model works as follows. We have an online minimization problem, an online algorithm ALG for the problem, and an adversary ADV for ALG. We introduce an oracle 0 that knows the whole input in advance. Before ALG starts its computation, 0 helps the algorithm by writing some binary information about the input on an infinitely large advice tape. During its computation, ALG may access the advice tape sequentially. The total number of the advice bits read in the worst case is the advice complexity of ALG.

Definition 4 (Online Algorithm with Advice [9]) Let I be an input of an online minimization problem, $I=\left(x_{1}, \ldots, x_{n}\right)$. Let OPT be an optimal solution for the corresponding offline problem. An online algorithm ALG with advice calculates a feasible output $\operatorname{ALG}^{\phi}(I)=\left(y_{1}, \ldots, y_{n}\right) \in \mathcal{O}(I)$ such that for $i \in$ $\{2, \ldots, n\}, y_{i}$ depends solely on $\phi, x_{1}, \ldots, x_{i}$ and on $y_{1}, \ldots, y_{i-1}$ ( $y_{1}$ depends solely on $\left.\phi, x_{1}\right)$, where $\phi$ is the content of the advice tape, i.e., an arbitrarily large binary sequence. ALG is c-competitive with advice complexity $b(n)$ if for every $n \in \mathbb{N}$ and for every input of length at most $n$, there exists some $\phi$ such that $\operatorname{cost}\left(\mathrm{ALG}^{\phi}(I)\right) \leq c \cdot \operatorname{cost}(\mathrm{OpT}(I))$ and at most the first $b(n)$ bits of $\phi$ have been read during the computation of $\mathrm{ALG}^{\phi}(I)$.

Although $b(n)$ depends on the input length $n$, we will write $b$ instead to keep our notation simple. So $b$ is the maximal number of bits accessed during the computation on an input instance of length $n$. We call the first $b$ bits of $\phi$ advice bits and say that ALG uses $b$ advice bits when ALG accesses $b$ bits of $\phi$ during its computation. In addition, we omit $\phi$ and write $\operatorname{Alg}(I)$ instead of $\operatorname{ALG}^{\phi}(I)$ as it is always clear from context.

The advice complexity of an online algorithm is an upper bound on the amount of information it needs in order to be $c$-competitive. We may consider online computation to be a game between the online algorithm ALG with advice, its adversary ADV, and the oracle 0 that works as follows [9, p. 25].

1. ADv knows ALG and 0 and $b$. ADV then constructs an instance $I$ such that the competitive ratio of ALG is maximized.

2. 0 examines $I$ and writes its advice $\phi$ on the advice tape.

3. Alg computes an output for $I$ using at most the first $b$ bits of $\phi$.

The following observation is crucial for our thinking about online algorithms with advice. Let ALG be an online algorithm with advice that reads $b$ advice bits. For a given instance $I$, ADV can treat ALG as a set of $2^{b}$ deterministic online algorithms without advice from which the algorithm with the lowest competitive ratio on the given input is chosen [9]. ADV knows each of the $2^{b}$ algorithms.

\subsection{Job Shop Scheduling with Unit Length Tasks}

The problem studied in this paper is called job shop scheduling, which is a widely applicable optimization problem. We are going to examine job shop scheduling 
with two jobs and unit-length tasks. Informally speaking, the problem can be described as follows. We have a factory with $m$ machines. Two customers arrive with one job each; each job wants to use each machine once and each wants to do this in a certain fixed order. In other words, a job consists of $m$ tasks and can be described as a permutation of the numbers $1, \ldots, m$ of the machines in the factory. A machine can process only one task at a time, and every machine needs one time unit to process a task. If, e.g., both customers want to use Machine 1 first, one of them has to wait. The goal is to minimize the cost, which is the total amount of time needed to process all tasks, also called the makespan.

Before we define the problem formally, we explain how we can represent it graphically, which was first made by Akers [1]. We take a grid of size $m \times m$. We assign coordinates to every intersection in the grid; the lower left corner has coordinates $(0,0)$; the top right corner has coordinates $(m, m)$. Such grids are shown in Figure 1. We label the $x$-axis with $\pi_{1}$ and the $y$-axis with $\pi_{2}$. Every square $(i, j)$ to $(i+1, j+1)$ of the grid that does not have

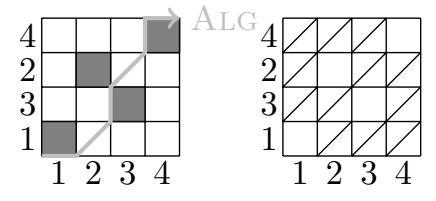

Fig. 1. Grid with obstacles on the left, grid with diagonals on the right. the same label on the $x$-axis as on the $y$-axis, i.e., where $\pi_{1}(i+1) \neq \pi_{2}(j+1)$, receives a diagonal edge from $(i, j)$ to $(i+1, j+1)$. Instead of drawing the diagonals, we often only draw the obstacles, i.e., the squares in the grid where there are no diagonal edges. An algorithm for this problem now starts at the lower left corner and moves on the edges of the grid to the top right corner. The cost of an algorithm equals the number of edges it uses.

Let us provide an example. Let $\pi_{1}=(1,2,3,4)$ and $\pi_{2}=(1,3,2,4)$ be the two jobs. Figure 1 depicts the grid and a solution computed by an algorithm ALG. With this picture in mind, we come to the formal definition.

Definition 5 (Job Shop Scheduling with Two Jobs, JSS) Let $m$ be a natural number, $m>0$. An input instance $I$ of JSS is of the form $\left(\pi_{1}, \pi_{2}\right)$, where $\pi_{1}$ and $\pi_{2}$ are permutations of $1, \ldots, m$. An output $O \in \mathcal{O}(I)$ consists of two injective functions $f_{1}, f_{2}:\{1, \ldots, m\} \rightarrow\{1, \ldots, 2 m\}$ that map the machines to time steps such that for $r, s \in\{1, \ldots, m\}$ :

1. $\forall i \in\{1,2\}: f_{i}\left(\pi_{i}(r)\right)<f_{i}\left(\pi_{i}(s)\right)$ if and only if $r<s$ (the order of the machines defined by the jobs has to be respected), and

2. $f_{1}(r) \neq f_{2}(r)$ (it is not possible to process two tasks on the same machine at the same time).

The cost of an algorithm ALG on an instance I is defined as the makespan, i.e., the time when the last task has been executed: $\max _{i \in\{1,2\}}\left\{f_{i}\left(\pi_{i}(m)\right)\right\}$.

We use the terms output and solution interchangeably. Moreover, when we take an instance $I$ of JSS, $m$ always denotes the number of machines. We will call the time units a solution needs to schedule all tasks steps; every solution 
for JSS needs at least $m$ steps. ${ }^{1}$ We call a step a diagonal step if both a task from job $\pi_{1}$ and a task from job $\pi_{2}$ is executed. Similarly, we call it a horizontal step (vertical step) when only a task from $\pi_{1}\left(\pi_{2}\right)$ is executed. The delay of a solution $\operatorname{Alg}(I)$ is defined as the number of non-diagonal steps divided by two; the delay of ALG on $I$ equals cost $\operatorname{ALG}(I))-m[8]$.

We will use diagonal strategies, which were introduced by Hromkovič et al. as a useful combinatorial tool [8, p. 9]. Let $I$ be an instance of JSS. By Diago we denote the main diagonal from the lower left corner to the top right corner of the grid. For $i \in\{1, \ldots, m-1\}$, Diag $_{i}$ is the diagonal from $(i, 0)$ to $(m, m-i)$, $i$ squares below Diag $_{0}$. For $i \in\{-1, \ldots,-(m-1)\}, \operatorname{Diag}_{i}$ is the diagonal from $(0, i)$ to $(m-i, m)$. To each diagonal $\operatorname{Diag}_{i}$, we assign an algorithm $\mathrm{D}_{i} \cdot \mathrm{D}_{i}$ first takes $i$ horizontal or vertical steps towards the starting point of $D_{i a g_{i}}$, i.e., for $i>0$ towards $(i, 0)$, for $i<0$ towards $(0, i)$. Then, $\mathrm{D}_{i}$ always takes diagonal steps until it reaches an obstacle. There, $\mathrm{D}_{i}$ takes a horizontal step directly followed by a vertical step, thus avoiding the obstacle before it continues with diagonal steps. It is not difficult to verify $\left[8\right.$, p. 9] that the cost of $\mathrm{D}_{i}$ on an instance $I$ equals the number of obstacles on $\operatorname{Diag}_{i}$ plus $m$ plus $|i|$.

\section{The New Model of Advice Complexity}

We study a modified model of advice complexity that introduces more powerful adversaries, which was established by Hromkovič [6]. This way, the oracle does not foresee the future exactly, which makes the setting more realistic. Indeed, in paging, for example, one can imagine that the algorithm knows which requests appear how frequently because of the typical behavior of the user. We observe the effects of our change with a strong focus on JSS. The game between the three parties online algorithm ALG with advice, oracle 0, and the adversary ADV, which is going to construct $d$ input instances, changes as follows:

1. As before, ADV knows the algorithm ALG and the oracle 0 .

2. Let $d \in \mathbb{N}$. ADV now constructs $\bar{d} \leq d$ input instances $I_{1}, \ldots, I_{\bar{d}}$ for ALG. We call $\mathcal{I}:=\left\{I_{1}, \ldots, I_{\bar{d}}\right\}$ the instance set.

3. ADV chooses a probability distribution $\rho$ over $\mathcal{I}$, i.e., for each input instance $I_{i}$, it chooses a probability $\rho\left(I_{i}\right)$, which we also denote by $\rho_{i}$.

4. 0 inspects $\mathcal{I}$ and $\rho$. 0 may then write some advice bits on the advice tape. Moreover, after each output of ALG, 0 may write some advice bits on the part of the advice tape not accessed by ALG. On each $I \in \mathcal{I}$, the content of the advice tape is the same at the beginning of ALG's computation.

5. On each $I \in \mathcal{I}$, Alg produces an output Alg $(I)$ depending on $I$ and the content of the advice tape.

6. The cost of Alg on $\mathcal{I}$ is defined as the expected cost, i.e., $\sum \rho_{i} \cdot \operatorname{cost}\left(\operatorname{ALG}\left(I_{i}\right)\right)$.

The reason why we allow 0 to write some advice after each output of ALG is that we want to include the fact that 0 does not know on which instance ALG

\footnotetext{
${ }^{1}$ By this, we mean that the output of every algorithm has a makespan larger than or equal to $m$.
} 
runs at the beginning of ALG's computation, but at some point, 0 recognizes the instance. At this point, it has to be possible for 0 to use its knowledge.

Definition 6 ( $\boldsymbol{d}$-Adversary and $\boldsymbol{d}$-Algorithm) Let $d \in \mathbb{N}$. We call an adversary that may construct at most $d$ input instances as above a d-adversary. An online algorithm "against" a d-adversary is called a d-algorithm.

Most of the time, we write $E_{\mathrm{ADV}}[\operatorname{cost}(\mathrm{ALG})]$ or even $E[\operatorname{cost}(\mathrm{ALG})]$ instead of $E_{\rho}[\operatorname{cost}(\mathrm{ALG})]$ if it is clear from the context which probability distribution or which adversary we mean.

Note that a 1-adversary in the new model corresponds to an adversary of the old model. Therefore, this model is a generalization of the old one. We know from the observation in Section 1 that ADV can treat ALG as a set of $2^{b}$ deterministic algorithms $\mathrm{ALG}_{1}$ to $\mathrm{ALG}_{2^{b}}$. The oracle 0 is going to choose the algorithm $\mathrm{ALG}_{i}$ with minimal competitive ratio. Therefore, ADV is going to take a set $\mathcal{I}$ and a probability distribution $\rho$ such that $\min _{i \in\left\{1, \ldots, 2^{b}\right\}} E_{\rho}\left[\operatorname{cost}\left(\mathrm{ALG}_{i}\right)\right] / E_{\rho}[\operatorname{cost}(\mathrm{OPT})]$ is maximized.

Some proofs in the remainder of this paper are omitted due to space constraints.

\section{Implications of the New Model}

First, observe that the new model is not easier in the sense that the cost and the competitive ratio of an online algorithm cannot improve when increasing the value $d$.

We now focus on $d$-adversaries in JSS. On the one hand, we will see in Corollary 8 that an algorithm based on an algorithm by Hromkovič et al. [8] reads a number of advice bits that is logarithmic in $m$ and achieves a competitive ratio of at most $1+1 / \sqrt{m}$. On the other hand, we show in Theorem 9 that there does not exist an optimal online algorithm with advice irrespective of the number of advice bits $b .^{2}$ In the old model, $2 \sqrt{m}$ advice bits were enough to achieve optimality [2]. We then prove that if $d$ is large enough, every online algorithm with advice has a competitive ratio of at least $1+1 /(6 \sqrt{m})$. Therefore, it seems as if the new model is similarly hard as the old model considering a logarithmic amount of advice bits; however, the new model is more difficult considering very large amounts of advice. Further research has to be carried out to understand how much harder the new model is for few advice bits.

We start by providing an upper bound on the expected cost of an online algorithm with advice. Recall that $m$ denotes the number of machines.

Theorem 7 Let DIAG be an algorithm that chooses a diagonal strategy upon reading $\log (2\lfloor\sqrt{m}\rfloor+1)$ advice bits; this algorithm is based on an algorithm from Hromkovič et al. [8]. Let $\mathrm{ADV}$ be any d-adversary. Then $E_{\mathrm{ADV}}[\operatorname{cost}(\mathrm{DIAG})] \leq$ $m+\lceil\sqrt{m}\rceil$.

\footnotetext{
$\overline{{ }^{2} \text { This is only }}$ true for $d$-adversaries with $d \geq 2$, of course.
} 
The idea of the proof is to sum the expected costs of all diagonal strategies $\mathrm{D}_{i}, i \in\{-\sqrt{m}, \ldots, \sqrt{m}\}$ and then to bound this sum from above by using that there are at most $m$ obstacles in each instance. Afterwards, the result is divided by $2 \sqrt{m}+1$ to obtain the average cost of a diagonal strategy.

Note that the upper bound on the cost of DIAG in Theorem 7 is essentially tight since there are instances for which an optimal solution OPT has $\operatorname{cost}(\mathrm{OPT}) \geq m+\lfloor\sqrt{m}\rfloor[8]$.

Corollary 8 Since the cost of any algorithm is at least $m$, Theorem 7 implies that DiAG has a competitive ratio of at most comp(DIAG) $\leq(m+\lceil\sqrt{m}\rceil) / m \leq$ $1+1 /\lfloor\sqrt{m}\rfloor$, which tends to 1 for $m$ going to infinity.

Being optimal against a $d$-adversary is not possible, as we can see in the following theorem.

Theorem 9 For all $m \geq 5$, there is a 2-adversary ADV such that no online algorithm ALG with advice can be optimal.

Note that if there is such a 2-adversary, then of course there is such a $d$-adversary for all $d>2$ as well. Moreover, the theorem holds irrespective of the number of advice bits ALG reads. Such an unlimited advice basically turns the problem into an offline problem.

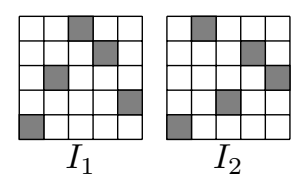

Fig. 2. The two instances of ADV.

Proof: For $m=5$, we define $I_{1}$ and $I_{2}$ as shown in Figure 2. For $m>5$, we fill up the rest of $I_{1}$ and $I_{2}$ with obstacles on the main diagonal. For the probabilities $\rho\left(I_{1}\right)$ and $\rho\left(I_{2}\right)$, we only need $\rho\left(I_{1}\right)>0$ and $\rho\left(I_{2}\right)>0$; for simplicity, we choose $\rho\left(I_{1}\right)=\rho\left(I_{2}\right)=\frac{1}{2}$.

Clearly, the cost of the only optimal solution Opt is $\rho\left(I_{1}\right)(m+1)+\rho\left(I_{2}\right)(m+$ $1)=m+1 .{ }^{3}$ Since the two first tasks are exactly the same for both instances, the oracle cannot know with which instance ALG deals. Therefore, before the first step, it provides the same advice on $I_{1}$ as on $I_{2}$; thus, ALG takes the same step for both $I_{1}$ and $I_{2}$, and we have

$$
E[\operatorname{cost}(\mathrm{ALG})] \geq \frac{1}{2} \cdot(m+2)+\frac{1}{2} \cdot(m+1)=m+\frac{3}{2}>E[\operatorname{cost}(\mathrm{OPT})],
$$

which concludes the proof.

We saw that we cannot be optimal. However, the proven difference in (1) between the cost of an online algorithm with advice and the cost of an optimal solution is rather small. We ask ourselves whether this is always the case. In other words, what is the best competitive ratio we can get?

In the following, we take the position of a $d$-adversary and create a class of instance sets on which every online algorithm with advice has a competitive ratio of at least $1+1 /(6 \sqrt{m})$. This result cannot be improved a great deal since we showed in Corollary 8 that the online algorithm DiAG with advice from Theorem 7 has a competitive ratio of at most $1+1 / \sqrt{m}$.

\footnotetext{
${ }^{3}$ Opt first takes a horizontal (vertical) step in $I_{1}\left(I_{2}\right)$, afterwards $m-1$ diagonal steps, and finally one vertical (horizontal) step.
} 
Preparations We proceed as follows. For some $d$, we act as a $d$-adversary. We take an instance set $\mathcal{I}=\left\{I_{1}, \ldots, I_{d}\right\}$ such that all instances have a common non-trivial prefix, i.e., there exists an $n>0 \in \mathbb{N}$ such that for any two instances $I_{i}=\left(\pi_{1}, \pi_{2}\right)$ and $I_{j}=\left(\sigma_{1}, \sigma_{2}\right)$, we have for all $i \leq n$ that $\pi_{1}(i)=\sigma_{1}(i)$ and $\pi_{2}(i)=\sigma_{2}(i)$. We take the equidistribution as the probability distribution $\rho$. For a fixed advice string, an online algorithm with advice will always behave the same in the prefix no matter what the rest of the instance looks like. We choose the tasks after the common prefixes such that independent of how an online algorithm with advice moves within the prefix, it is optimal in at most one instance and it has certain additional costs in all other instances.

Let $k \geq 3 \in \mathbb{N}$. Let $J=\left(\sigma_{1}, \sigma_{2}\right)$ be the hard instance of size $\left(k+\left\lceil\frac{k}{2}\right\rceil\right)^{2}$ constructed by Hromkovič et al. [8, p. 6]. An example for a hard instance of size $5^{2}$ is given in Figure 3. Every algorithm for $J$ has a cost of at least $m+k+\left\lceil\frac{k}{2}\right\rceil$ [8]. We take $k+1$ copies of $J$ and denote them by $I_{1}, \ldots, I_{k+1}$. After some modification, all instances are going to be pairwise different and we then define $\mathcal{I}=\left\{I_{1}, \ldots, I_{k+1}\right\} .{ }^{4}$ We choose for each instance $I_{i}$ a coordinate point $\left(x_{i}, y_{i}\right)$ and then modify the instances such that for each instance $I_{i}$

1. the first $\left(k^{2}+k\right) / 2$ tasks of each job remain the same,

2. all optimal solutions pass $\left(x_{i}, y_{i}\right)$,

3. all solutions that do not pass $\left(x_{i}, y_{i}\right)$ have additional cost (compared to an optimal solution) depending on "how far away" they pass $\left(x_{i}, y_{i}\right)$, and

4. $\left(x_{i}, y_{i}\right) \neq\left(x_{j}, y_{j}\right)$ for all $I_{j} \neq I_{i}$.

We consider the diagonal that crosses all coordinates $\left(i, k^{2}-i\right)$ with $i \in\left\{0, \ldots, k^{2}\right\}$, which is a diagonal orthogonal to Diag. . We call this diagonal slot diagonal. All intersections on the grid that lie on this diagonal are called slots. An example for $k=3$ is shown in Figure 3. Clearly, every algorithm passes exactly one slot.

We have $k+1$ slots that are next to an obstacle, namely all the slots with coordinates $\left(i, k^{2}-i\right)$ for $i \in$ $\left\{\frac{k^{2}-k}{2}, \ldots, \frac{k^{2}+k}{2}\right\}$. From left to right, we enumerate these slots from 1 to $k+1$, e.g., slot 1 is the slot with coordinates $\left(\frac{k^{2}-k}{2}, \frac{k^{2}+k}{2}\right)$. For $i \in\{1, \ldots, k+$ $1\}$, slot $i$ is going to be the coordinate point $\left(x_{i}, y_{i}\right)$ for $I_{i}$ from our list above.

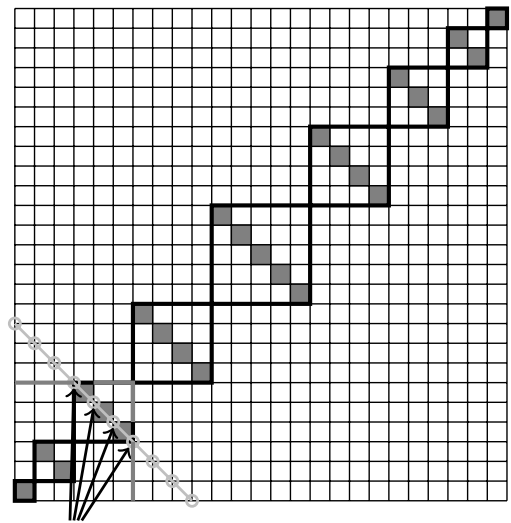

Slots 1 to 4

Fig. 3. An instance for $k=3$ with the slot diagonal and the slots in lightgray. The upper and the right border of the common prefix are marked with gray.

We impose a distance function, $\operatorname{dist}(\cdot, \cdot)$, on the slots. We take the intuitive distance between two slots; i.e., the distance between two slots equals the number

\footnotetext{
${ }^{4}$ Thus, $d=k+1=2 \sqrt{m} / 3+1$ if $k$ is even and $d=k+1=2 \sqrt{m} / 3+2 / 3$ if $k$ is odd.
} 
of slots between them plus 1 , the distance from a slot to itself is defined as 0 . This distance is the "how far away" from the list above.

Trivially, every algorithm has to make at least $|i|$ horizontal steps or $|i|$ vertical steps to reach a slot on $\operatorname{Diag}_{i}$. For the slots 1 to $k+1$, we have the following lemma. Recall that $J=\left(\sigma_{1}, \sigma_{2}\right)$ is the hard instance for JSS of size $\left(k+\left\lceil\frac{k}{2}\right\rceil\right)^{2}$.

Lemma 10 Let $I=\left(\pi_{1}, \pi_{2}\right)$ be an instance of size $\left(k+\left\lceil\frac{k}{2}\right\rceil\right)^{2}$ such that for $i \in\left\{1, \ldots,\left(k^{2}+k\right) / 2\right\}$, the tasks $i$ of $I$ and $J$ equal, i.e., $\sigma_{1}(i)=\pi_{1}(i)$ and $\sigma_{2}(i)=\pi_{2}(i)$. For $i \in\{1, \ldots, k+1\}$, every algorithm for $I$ has to make at least $k+1-i$ vertical steps and at least $i-1$ horizontal steps to reach slot $i$. Moreover, there exists an algorithm that achieves this amount of steps.

The idea of the proof is to use induction.

Construction of Instances We now modify the instances $I_{1}, \ldots, I_{k+1}$. Take any instance $I_{i}=\left(\pi_{1}, \pi_{2}\right)$. We want to ensure that once an algorithm that takes a diagonal step whenever possible reaches slot $i$, there are no more obstacles in its way. Denote the diagonal on which slot $i$ is by Diag $g_{q}$. Consider all obstacles that are on $\operatorname{Diag}_{q}$ after slot $i$. Our goal is to remove them from Diag . Denote the number of such obstacles by $z$. We know that $z \geq 2$ since we consider hard instances of size $\left(k+\left\lceil\frac{k}{2}\right\rceil\right)^{2}$ and $k \geq 3$. We discuss the case when $z$ is even first. We take $z / 2$ times two obstacles and switch the two tasks of Job 2. We show in Figure 4 what we do.

We describe what happens exactly. An obstacle on Diag $_{q}$ corresponds to a square in the grid where the task of Job 1 and the task of Job 2 equal, i.e., there is a Machine $a$ such that $\pi_{1}(x)=$ $a=\pi_{2}(y)$ with $x-y=q$. We have another obstacle on $\operatorname{Diag}_{q}$, i.e., there is a Machine $b \neq a$ such that we have

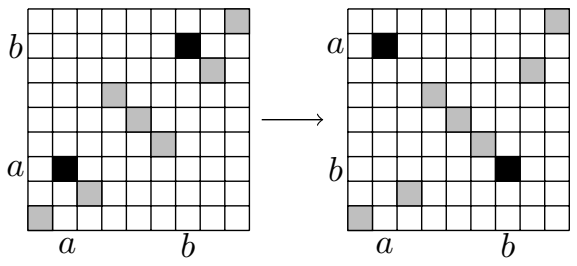

Fig. 4. Switching two tasks. two tasks $\pi_{1}(v)=b=\pi_{2}(w)$ with $v-w=q$. If the second obstacle comes after the first, we have $x<v$ and $y<w$. We switch tasks $\pi_{2}(y)=a$ and $\pi_{2}(w)=b$, i.e., we define $\pi_{2}(y)=b$ and $\pi_{2}(w)=a$. In other words, if the $y$-th task of Job 2 was before Machine $a$ and the $w$-th task of Job 2 was before Machine $b$, now the $y$-th task of Job 2 is Machine $b$ and the $w$-th task of Job 2 is Machine $a$. Note that this process does not affect the position of any other obstacle.

We have to ask on which diagonals the new obstacles lie. Assume towards contradiction that the new obstacles lie on $\operatorname{Diag}_{q}$. The new obstacles are $\pi_{1}(x)=$ $a=\pi_{2}(w)$ and $\pi_{1}(v)=b=\pi_{2}(y)$; hence, the new obstacles are on diagonals $x-w$ and $v-y$, and we have

$$
x-w=q \Longleftrightarrow x-(v-q)=q \Longleftrightarrow x=v,
$$

which is a contradiction to the fact that we chose two different obstacles. An analogous calculation is true for $v-y$, so the new obstacles are indeed not on 
$\operatorname{Diag}_{q}$, and when we switch the tasks of all $z / 2$ pairs of obstacles, there are no more obstacles on $\operatorname{Diag}_{q}$ after slot $i$.

We now come to the case when $z$ is odd. We have that $z \geq 3$. We take $(z-1) / 2-2 \geq 0$ times two obstacles and switch their tasks of Job $\overline{2}$ as described above. There are $z-2((z-1) / 2-2)=3$ remaining obstacles. The corresponding labels are $\pi_{1}(x)=a=\pi_{2}(y), x-y=q ; \pi_{1}(v)=b=\pi_{2}(w), v-w=q$; and $\pi_{1}(s)=c=\pi_{2}(t), s-t=q$, where we chose the order such that $x<v<s$ and $y<w<t$. We switch their tasks of Job 2 as follows: $\pi_{2}(y)=c, \pi_{2}(w)=a$, $\pi_{2}(t)=b$. Again, the new obstacles do not lie on $\operatorname{Diag}_{q}{ }^{5}$

Cost of an Optimal Solution By changing the obstacles as described above, we have ensured for all $i \in\{1, \ldots, k+1\}$ that once a solution $\mathrm{OPT}_{i}$ for $I_{i}$ that takes a diagonal step whenever possible passes slot $i$, it does not encounter any further obstacles. We calculate the cost of such an $\mathrm{OPT}_{i}$. According to Lemma 10 , there exists an optimal solution $\mathrm{OPT}_{i}$ that makes $i-1$ horizontal steps and $k+1-i$ vertical steps in order to pass slot $i$. We let $\mathrm{OPT}_{i}$ take a diagonal step whenever possible from slot $i$ onwards; as a consequence, $\mathrm{OPT}_{i}$ does not meet any obstacles once it has passed slot $i$. Therefore, the delay of $\mathrm{OPT}_{i}$ is $\max \{i-1, k+1-i\}$. We will prove later that the cost of any solution that does not pass slot $i$ is higher than the cost of $\mathrm{OPT}_{i}$. Together with the fact that $\mathrm{OPT}_{i}$ has minimal cost among all the solutions passing slot $i$, it follows that $\mathrm{OPT}_{i}$ is indeed optimal for $I_{i}$.

Given that for all $i \in\{1, \ldots, k+1\}, \mathrm{OPT}_{i}$ is optimal for $I_{i}$, we have an optimal solution Opt for $\mathcal{I}$ by taking $\mathrm{OPT}_{i}$ on $I_{i}$. We want to calculate the expected cost of OpT over all instances $I_{i}$. We only provide the calculations for the case where $k$ is even. We calculate for $k$ even the total cost of OPT.

$$
\sum_{i=1}^{k+1} \operatorname{cost}\left(\operatorname{OPT}_{i}\left(I_{i}\right)\right)=\sum_{i=1}^{k+1} m+\max \{i-1, k+1-i\}=(k+1) m+\frac{3}{4} k^{2}+k .
$$

Dividing the result by $k+1$, we obtain an expected cost of

$$
m+\frac{3}{4} k+\frac{1}{4}-\frac{1}{4(k+1)} \text {. }
$$

Cost of a Non-Optimal Solution We want to calculate the delay of any solution ALG on $I_{i}$ and compare this delay to the delay of $\mathrm{OPT}_{i}$. Note first that

$$
i-1 \geq k+1-i \Longleftrightarrow 2 i-k-2 \geq 0 \Longleftrightarrow i \geq \frac{k}{2}+1
$$

\footnotetext{
${ }^{5}$ Note that it does not matter for our calculation how we pick the pairs (and maybe the triple) of obstacles. If, e.g., $z=4$, it does not matter if we switch the tasks of the first and the second obstacle and the tasks of the third and the forth obstacle or if we switch the tasks of the first and the third obstacle and the tasks of the second and the forth obstacle. To ensure that the modification is well-defined, we always take pairs of obstacles that are next to each other, e.g., if $z=4$, we pair obstacle 1 with obstacle 2 and obstacle 3 with obstacle 4 . If $z$ is odd, we take the three obstacles at the end to switch the three tasks.
} 
Lemma 11 Let ALG be a deterministic solution for $I_{i}$ that passes the slot diagonal on a slot $(h, v)$ with distance $j \geq 1$ to slot $i$. Then we have

$$
\operatorname{cost}\left(\operatorname{ALG}\left(I_{i}\right)\right) \geq \min \left\{\operatorname{cost}\left(\mathrm{Opt}_{i}\left(I_{i}\right)\right)+j, \operatorname{cost}\left(\mathrm{Opt}_{i}\left(I_{i}\right)\right)+\left\lceil\frac{k}{2}\right\rceil\right\} .
$$

The idea of the proof is to make a case distinction on the slot which ALG passes.

Now we want to analyze the behavior of an online algorithm with advice ALG on $\mathcal{I}$. On any instance $I_{i}$, ALG behaves the same on the common prefix. We divide the border of the prefix into three regions and estimate the cost of ALG depending on where it reaches the border. The algorithm ALG reaches the prefix at exactly one of these regions. Region 1 is at the upper border of the prefix and consists of all coordinates $\left(x, \frac{k^{2}+k}{2}\right)$ with $x \in\left\{0, \ldots, \frac{k^{2}-k}{2}\right\}$. Region 2 is at the right border of the prefix and consists of all coordinates $\left(\frac{k^{2}+k}{2}, x\right)$, $x \in\left\{0, \ldots, \frac{k^{2}-k}{2}\right\}$. Region 3 consists of the remaining coordinates on the upper and the right border of the prefix, i.e., of all coordinates $\left(x, \frac{k^{2}+k}{2}\right)$ and of the coordinates $\left(\frac{k^{2}+k}{2}, x\right)$ with $x \in\left\{\frac{k^{2}-k}{2}+1, \ldots, \frac{k^{2}+k}{2}\right\}$.

It turns out that ALG minimizes its cost by reaching the border of the prefix at Region 3. More precisely, for $k$ even, slot $k / 2$ minimizes the cost; for $k$ odd, both slot $\lfloor k / 2\rfloor$ and slot $\lceil k / 2\rceil$ minimize the cost. If ALG chooses slot $k / 2$, it has at least total cost of

$$
\sum_{i=1}^{k+1} \operatorname{cost}\left(\operatorname{OPT}_{i}\left(I_{i}\right)\right)+2 \sum_{i=1}^{k / 2} i=\sum_{i=1}^{k+1} \operatorname{cost}\left(\operatorname{OPT}_{i}\left(I_{i}\right)\right)+\frac{k^{2}}{4}+\frac{k}{2} .
$$

Together with (2), we see that this is equal to

$$
(k+1) m+\frac{3}{4} k^{2}+k+\frac{k^{2}}{4}+\frac{k}{2}=(k+1) m+k^{2}+\frac{3}{2} k .
$$

Lower Bound on Competitive Ratio For $k$ even, we use (5) to compute the expected cost of an online algorithm with advice ALG that takes slot $k / 2$ :

$$
E[\operatorname{cost}(\operatorname{ALG}(\mathcal{I}))]=\frac{\text { total cost of ALG }}{k+1}=m+k+\frac{1}{2}-\frac{1}{2(k+1)} .
$$

Together with (3) and the fact that $m=9 k^{2} / 4$, we have a competitive ratio of at least

$$
\begin{aligned}
\frac{m+k+\frac{1}{2}-\frac{1}{2(k+1)}}{m+\frac{3}{4} k+\frac{1}{4}-\frac{1}{4(k+1)}} & =\frac{m+\frac{3}{4} k+\frac{1}{4}-\frac{1}{4(k+1)}+\frac{1}{4} k+\frac{1}{4}-\frac{1}{4(k+1)}}{m+\frac{3}{4} k+\frac{1}{4}-\frac{1}{4(k+1)}} \\
& \geq 1+\frac{\frac{1}{4}(k+1)}{\frac{9}{4} k(k+1)}=1+\frac{1}{6 \sqrt{m}} .
\end{aligned}
$$


This finishes the proof. We showed that for all $m=(k+\lceil k / 2\rceil)^{2} \geq(3+2)^{2}$, there is a $d$-adversary, $d=2 \sqrt{m} / 3$ if $k$ is even and $d=2 \sqrt{m} / 3+2 / 3$ if $k$ is odd, such that no online algorithm ALG with advice has a competitive ratio smaller than $1+1 /(6 \sqrt{m})$.

\section{Acknowledgements}

I want to thank Juraj Hromkovič for his useful advice and support; Dennis Komm for his effort in giving this work impetus and the many hours he spent with proofreading; and Hans-Joachim Böckenhauer for many helpful discussions.

\section{References}

1. S. B. Akers. A graphical approach to production scheduling problems. In Operations Research, 4(2):244-245, Informs, 1956.

2. H.-J. Böckenhauer, D. Komm, R. Královič, R. Královič, and T. Mömke. On the advice complexity of online problems. In Algorithms and Computation (ISAAC 2009, volume 5878 of Lecture Notes in Computer Science, pages 331-340, SpringerVerlag, 2009.

3. A. Borodin and R. El-Yaniv. Online Computation and Competitive Analysis. Cambridge University Press, 1998.

4. S. Dobrev, R. Královič, and D. Pardubská. How much information about the future is needed? In Theory and Practice of Computer Science (SOFSEM 2008), volume 4910 of Lecture Notes in Computer Science, pages 247-258. Springer-Verlag, 2008.

5. Y. Emek, P. Fraigniaud, A. Korman, and A. Rosén. Online computation with advice. Theoretical Computer Science 412(24):2642-2656, 2011.

6. J. Hromkovič. New model of advice complexity. Personal communication, 2013.

7. J. Hromkovič, R. Královič, and R. Královič. Information complexity of online problems. In Mathematical Foundations of Computer Science (MFCS 2010), volume 6281 of Lecture Notes in Computer Science, pages 24-36. Springer-Verlag, 2010.

8. J. Hromkovič, T. Mömke, K. Steinhöfel, and P. Widmayer. Job shop scheduling with unit length tasks: Bounds and algorithms. In Theoretical Computer Science (ICTCS 2001), volume 2202 of Lecture Notes in Computer Science, pages 90-106, Springer-Verlag, 2001.

9. D. Komm. Advice and randomization in online computation. Dissertation at ETH Zürich No. 20164, 2012.

10. D. Komm and R. Královič. Advice Complexity and Barely Random Algorithms. In Proc. of the 37th International Conference on Current Trends in Theory and Practice of Computer Science (SOFSEM 2011), volume 6543 of Lecture Notes in Computer Science, pages 332-343, Springer-Verlag, 2011.

11. D. Komm and R. Královič. Advice Complexity and Barely Random Algorithms. RAIRO - Theoretical Informatics and Applications, 45(2):249-267. 2011.

12. D. D. Sleator and R. E. Tarjan. Amortized efficiency of list update and paging rules. In Communications of the ACM, 28(2):202-208, ACM, 1985. 